\title{
Aportes de la Escuela Fenomenológica y la Teoría Crítica a la Comprensión de la Problemática Hídrica dentro del Escenario Escolar
}

\section{Contributions of the Phenomenological School and Critical Theory to the Understanding of Water Problems within the School Setting}

\author{
Elisabeth Suárez Cerón \\ Licenciada en Ciencias Sociales, Trabajador Social, Especialista en Estudios Latinoamericanos, \\ Magíster en Educación y doctoranda en Ciencias de la Educación. Docente de tiempo completo en la \\ I.E. Escuela Normal Superior San Carlos de la Unión - Nariño. \\ Email: elizuce@yahoo.com
}

Doi: https:// https://doi.org/10.22267/rceilat.214849.94

\section{Resumen}

La problemática ambiental centrada en el recurso hídrico es susceptible de ser analizada bajo cualquier escuela de pensamiento; no obstante, abordarla desde la orientación fenomenológica, otorga la virtud de descubrir las claves que ayudan a descifrar, interpretar y actuar sobre la naturaleza de las causas que enquistan y alimentan este fenómeno. En este sentido, la escuela fenomenológica emerge como el olimpo epistemológico que vierte su iluminación sobre la problematización y discernimiento de un asunto, dentro del cual, los aportes de la teoría crítica y de eruditos posmodernos como Boaventura de Sousa, Habermas, Foucault y Morín, permiten alinear sus pensamientos para dar fuerza a los argumentos en torno a los cuales se posibilita procesar los hechos que le acontecen a la esfera ambiental, actualmente estropeada por un modo de producción y de organización social, cuya naturaleza destructiva, ha dejado profundas huellas en el deterioro de los recursos hídricos. Esta problemática, que constituye una preocupación de diversos ámbitos, también inquieta al campo educativo, por lo que desde este trabajo se entiende, que articular herramientas como el Proyecto Ambiental Escolar - PRAE y el Aprendizaje Basado en Problemas - ABP, facilita el desarrollo de procesos de enseñanza - aprendizaje, integradores de la realidad contextual con la cotidianidad del aula.

Desde el punto de vista metodológico, la construcción del documento se llevó a cabo utilizando el análisis bibliográfico.

Palabras claves: Aprendizaje Basado en Problemas - ABP; Fenomenología; Educación; Proyecto Ambiental Escolar - PRAE; Teoría socio crítica. 


\begin{abstract}
The environmental problem centered on the water resource is susceptible to be analyzed from any school of thought; However, approaching it from the phenomenological orientation gives the virtue of discovering the keys that help to decipher, interpret and act on the nature of the causes that encyst and feed this phenomenon. In this sense, the phenomenological school emerges as the epistemological Olympus that pours its illumination on the problematization and discernment of an issue, within which, the contributions of critical theory and postmodern scholars such as Boaventura de Sousa, Habermas, Foucault and Morín, allow you to align your thoughts to give strength to the arguments around which it is possible to process the events that happen to the environmental sphere, currently spoiled by a mode of production and social organization, whose destructive nature has left deep traces on the environment. Deterioration of water resources. This problem, which is a concern of various fields, also worries the academic field, so from this work it is understood, that resorting to tools such as the School Environmental Project PRAE and Problem-Based Learning - PBL, facilitates the development of processes of teaching - learning, integrators of the contextual reality with the daily life of the classroom.
\end{abstract}

Keywords: Phenomenology; Socio-critical theory; School Environmental Project; Educatión; Problem-Based Learning.

\section{Introducción}

La fenomenología que emerge como partera de la comprensión de las experiencias de los sujetos asociadas a un fenómeno, deviene de procesos metacognitivos discernidos por la conciencia y que desde el punto de vista social, configuran la esencia de las experiencias colectivas y facilita elementos epistemológicos, cuya lógica conduce a desentrañar las estructuras, escarbar las causas, analizar las consecuencias y proyectar alternativas autónomas que reorienten los impactos del fenómeno en términos satisfactorios para el orden social.

Por su parte, en lo concerniente al modelo socio - critico, es preciso recordar que su estructura paradigmática se consolida como contestación a la tradición positivista e interpretativa que se había mostrado incapaz de pro- mover la emancipación del ser humano; en consecuencia, surge como un enfoque que privilegia la comprensión de los hechos sociales que germinan en las estructuras de la cotidianidad, al igual que de los diversos mecanismos a través de los cuales lo habitual es digerido e incorporado en el comportamiento de cada individuo que integra la estructura social, generalmente caracterizada por la esencia interactiva de los sujetos.

De esta manera, la fenomenología y la teoría socio - crítica asociadas a la educación, constituyen una triada beneficiosa para reconstruir, retroalimentar, descartar, reorientar o validar los conocimientos y prácticas asociadas al manejo que hace la sociedad moderna de los recursos naturales, especialmente en lo relacionado a los hábitos y percepciones aplicadas al tema hídrico, respecto al cual, estra- 
tegias como el Proyecto Ambiental Escolar - PRAE y el Aprendizaje Basado en Problemas - ABP, motivan prácticas que facilitan la transformación del escenario pedagógico y en consecuencia de los espacios permeados por el hecho educativo. Las referidas ideas son las que se consideran y desarrollan a través del recorrido teórico que alimenta al presente ensayo.

La fenomenología, como escuela que surgió de la preocupación por explicar la naturaleza de los hechos, ofrece recursos teóricos para aprehender la realidad, derivar explicaciones, elaborar interpretaciones y abrir posibilidades hacia la construcción de nuevas percepciones que ahonden en la producción de conocimiento respecto a los múltiples objetos susceptibles de ser intervenidos por la natural curiosidad humana.

Pero esta tarea, exige del investigador una metódica labor exploradora, que después de introducirlo en el objeto de estudio y de escarbar en sus estructuras y esencias, le conlleve a proponer una narrativa de los hechos que simplemente descubra, detalle, devele, lo estimado indudable, sin alterar su naturaleza y en consecuencia preservando aquello que según la filosofía husserliana, es inmutable en el fenómeno, su esencia; esencia cuya interpretación es inherente a la conciencia del sujeto humano. En este ambiente, el investigador requiere asumir una postura crítica frente a la realidad, actitud ésta que necesariamente debe estar atravesada por la inquietud, la interrogación, el asombro, o lo que Husserl llama la duda, que la persona en su libre albedrío, está en la disposición de practicar de cara a una realidad espacio temporal que lo permea y está ahí, en estado natural, como pidiendo ser descubierta por la actitud de aquel, que agudizando sus sentidos y en estado de búsqueda y meditación, devele lo que es sensible a su conciencia, lo que percibe como objeto de indagación, de cuestionamiento, de deconstrucción, de búsqueda de discernimiento y de significado; es decir, que desde este oficio, siempre habrá lugar para bisoñas lecturas que aporten nuevos ángulos y dimensiones referidas a la entidad del objeto. (Lozano, 2006).

En este sentido, la búsqueda de tal precisión identitaria del objeto es lo que incomoda a los predicadores de verdades eternas y a algunas escuelas de pensamiento que precedieron a la fenomenología (el positivismo, por ejemplo), pero también es lo que genera pretextos para que la ciencia se interrogue por esos resquicios que no permiten que las piezas constituyentes de un objeto, de una versión o de un conocimiento calcen a la perfección. He ahí la incidencia de la capacidad de asombro, de curiosidad y de método, para atreverse a abrir la caja de pandora, que con mucha rigurosidad manipulan los científicos, filósofos e investigadores sociales, cuya pericia ha sido marcada por procesos educativos que constantemente motivan la percepción de las experiencias vitales con cierta inquisición. Tal habilidad que tras el ejercicio mecánico se torna natural, se constituye en partera de in- 
mensurables posibilidades creadoras de saberes que se forjan a partir del lente perceptivo de cada conciencia como parte constitutiva del mundo de las experiencias.

Ahora, el resultado de esas significaciones otorgadas a las experiencias, inevitablemente va estar cruzado por la huella indeleble que deja en el sujeto su paso por el sistema educativo, en cuyo seno se forjan vivencias colectivas que pasan a ser compartidas por los conglomerados que participan de ciertas realidades. En este ambiente, es donde se exteriorizan las cavilaciones, las vivencias intencionales de los sujetos, en forma de iniciativas o argumentos que son el reflejo de la suma de experiencias que han pasado los filtros de la razón, para luego concretarse en contenidos tangibles, que en la práctica dan lugar a habitar la experiencia de las verdades que aportan y comparten los sujetos. (Lambert, 2006) y (Anzola, 2007).

En este orden de ideas, para el interés de este trabajo, es preciso tomar de la fenomenología términos clave como Epojé, inmanencia e intencionalidad. Epojé como sinónimo de reducción y que (Lambert, 2006) lo asocia con "el acto de limitarse al examen de las puras vivencias como tales" (p.521). Este estado de la conciencia donde el juicio se suspende y por tanto solo hay espacio para absorber información, para tantear los hechos, para evaluar lo percibido, constituye la antesala de la inmanencia que, de acuerdo con el referido autor (2006), es el producto que se obtiene a partir del flujo de la con- ciencia que se materializa en "el conocimiento absoluto alcanzado en la reflexión... [proceso] que sirve de base para la teoría del conocimiento, pues gracias a ella el conocimiento se ve libre de todos los enigmas que atañen al ser trascendente del objeto conocido" (p. 522). De igual modo, en el umbral del tránsito de la epojé a la inmanencia, la intencionalidad irrumpe como energía esencial que le da cuerpo a las vivencias cognoscitivas y por tanto tal actividad, canaliza la relación entre la conciencia y el mundo. Es decir, la intencionalidad es la que activa el juicio en suspensión y hace que broten sus productos hacia el mundo exterior. La habilidad metódica de examinar las vivencias, reflexionarlas y descubrir su intencionalidad, fácilmente podría articularse a procesos pedagógico - investigativos, que redunden en la exploración de la conciencia como punto de partida para transformar las experiencias individuales y en efecto la vida en sociedad.

Estas disertaciones husserlianas, aunque ya superaron el siglo de existencia, alimentaron una escuela de pensamiento cuya esencia sigue vigente en el alma de paradigmas como la teoría crítica, dado que desde aquel manantial ideológico se forjó una doctrina que, según Ropero, (2019), orienta la "comprensión crítica de nuestro presente sociocultural”. (p. 119).

Así, la crítica del conocimiento, que obra a través de la práctica de la reducción fenomenológica, queda amparada en los sustentos filosófico - metodológicos de las corrientes de 
pensamiento más representativas del siglo $\mathrm{XX}$, que en términos del propio Husserl (cfr. 2012, p. 1-12) citado por Ropero (2019), abogan por la “«auténtica humanidad», por una «verdadera cultura racional», por los «valores universales» de la «autonomía» y la «libertad», tanto individual como colectiva" (p. 126); es decir, los principios Husserlianos respaldan una filosofía con compromiso social, en la que se reconoce a los individuos, "como sujetos de creencias, voliciones y tomas de partido ante los conflictos y los valores en pugna y, por tanto, como libres, responsables y «genuinamente morales»" (Ibid. p. 126), rasgos éstos perfectamente identificables en la teoría crítica.

Bajo este marco epistemológico, la teoría crítica, de descendencia fenomenológica, que emergió para perturbar el ordenamiento humano positivista, para agitar las contradicciones subyacentes a lo inmutable, para develar las fricciones discursivas presentes en la basta arquitectura social delineada por los intereses capitalistas, se constituye en el dispositivo teórico preciso para escudriñar las razones sustantivas que subyacen a las problemáticas sociales de tan diversos contextos y matices y, cuyos niveles de complejidad exigen integrar distintos actores y componentes. Son tan variados los problemas contenidos en el mundo de la vida y que están al alcance de la teoría crítica, que la realidad, en sus diversas facetas, es considerada como un escenario de variadas posibilidades que incluso trascienden la dimensión práctica. Esa esfera de lo huma- no prolija en complejidades, a parte de lo empírico también se expresa en reacciones de malestar, indignación e inconformismo en torno a situaciones críticas, que una vez consideradas sirven como pretexto para disertar y teorizar frente a presupuestos que orientan alternativas viables para mejorar las problemáticas. De Sousa, (2006).

Lo interesante de estas posturas es la evidente conexión que naturalmente emerge entre sujeto y objeto de estudio, fruto del proceso autorreflexivo que se plantea el investigador afectado por el estado de cosas que discurren en los escenarios cotidianos. En dichas experiencias la teoría crítica tiene lugar a formalizar esos conocimientos que brotan de los intereses formados en las necesidades de los conglomerados y donde la acción del teórico aparece articulada a la comprensión e incluso a la solución de los dilemas. Con relación a este apunte, De Sousa, (2006), establecerá que:

Nadie ha definido la teoría crítica moderna de una manera más adecuada que Max Horkheimer. La teoría crítica moderna es, sobre todo, una teoría epistemológicamente fundada en la necesidad de superar el dualismo burgués entre el científico individual como creador autónomo de conocimiento y la totalidad de la actividad social que lo rodea.

Mas adelante, el citado sociólogo, retoma una nota del propio Horkheimer, que reza:

La razón no se puede convertir en algo transparente a sí misma, mientras que el ser humano actúe como miembro de 
un organismo que carece de razón. La irracionalidad de la sociedad moderna reside en el hecho de que dicha sociedad ha sido producto de una voluntad particular, la del capitalismo, y no de una voluntad general, una voluntad mancomunada y consciente de sí misma. De Sousa, (2006, p.19)

Estas aportaciones que esclarecen los rasgos y objetivos de la teoría crítica, ponen al lector en sintonía con la utilidad de este paradigma, para analizar los fenómenos socio económicos e histórico culturales propios de las sociedades capitalistas, que actúan bajo la lógica del progreso ilimitado, la competencia irracional, el individualismo, la acumulación desmedida, la privatización de los servicios públicos, el consumismo, la terciarización laboral, la paradójica depredación de los recursos naturales, entre otras banderas del neoliberalismo capitalista, cuyo coletazo en los sectores poblacionales pauperizados, se expresa en índices crecientes de miseria, deserción escolar, delincuencia común, desempleo, descomposición social, ampliación de la frontera agrícola hacia zonas protegidas y la profundización de los riegos ambientales, para quienes, por las inequidades del modelo económico, son empujados a asentarse en áreas propensas a inundaciones, deslizamientos y sequias.

Las mencionadas realidades no son menos importantes de lo que se piensa, si se perciben desde una óptica de equilibrio y armonía entre la sociedad y entre ésta y la naturaleza; sin embargo, en el contexto de la pujante irracionalidad neoliberal, estos hechos están lejos de desaparecer, dado que es un modelo bien apalancado por las fuerzas políticas tradicionales, la banca y los medios de comunicación, empeñados en la tarea de menguar las dialécticas que se derivan de estas realidades, al tiempo que desde la razón instrumental, fortalecen regímenes de la verdad, copiados de la sociedad occidental, en donde ahora mismo, hay sectores desconfiados de sus éxitos, dados los evidentes resultados que amenazan en forma apocalíptica el mundo de la vida.

En este sentido, los principios que subyacen al paradigma socio crítico le confieren idoneidad para asumir situaciones que generan choques entre los diferentes factores que anidan en aquellos ámbitos donde la acción humana hace presencia, pues de acuerdo con Alvarado, y García, (2008), estudiosos de Popkewitz (1988), el enfoque crítico se fundamenta en preocupaciones que analizan la realidad $y$ junto a ese examen se proponen transformaciones. Las preocupaciones referidas son:

(a)conocer y comprender la realidad como praxis; (b) unir teoría y práctica integrando conocimiento, acción y valores; (c) orientar el conocimiento hacia la emancipación y liberación del ser humano; y (d) proponer la integración de todos los participantes, incluyendo el investigador, en procesos de autorreflexión y de toma de decisiones consensuadas, las cuales se asumen de manera corresponsable. Alvarado, y García, (2008 p. 190).

Estas virtudes del paradigma lo 
dotan de herramientas teórico prácticas para comprender las distintas tensiones y sacarles provecho, en la medida que las crisis para el enfoque socio-crítico, se asumen como oportunidades de mejoramiento para las cuales, los actores del cambio necesitan tener apertura para reconocer el conflicto como una parte necesaria de la situación, para promover las transformaciones que se buscan. De este modo, siendo que lo ambiental y específicamente el recurso hídrico, conforman temas que no pasan desapercibidos en las actuales agendas político económico de los dirigentes mundiales (porque justamente se sitúan como determinantes del desarrollo de la mayoría de las actividades socio económicas), estos mismos ejes, adquieren un tinte protagónico en los escenarios donde se debate y decide su futuro como elementos aislados de las comunidades y que por lo mismo generan desencuentros entre las medidas político económicas y las dinámicas propias de las gentes que naturalmente han apropiado dichos recursos. Esos desencuentros son los que revelan los desencantos de la modernidad, advertidos por De Sousa, (2006) en la perversa dominación que ha practicado el capitalismo sobre la naturaleza, desencadenando y sosteniendo la crisis ecológica que es de dominio público. Esta denuncia, el referido crítico, la respalda en los siguientes datos:

En los últimos 50 años el mundo ha perdido alrededor de una tercera parte de su reserva forestal. A pesar de que las selvas y los bosques tropicales proveen el $42 \%$ de biodiversidad y de oxígeno, 242.820 hec- táreas de reserva forestal mexicana han sido destruidas cada año. Hoy día las empresas multinacionales tienen el derecho de talar árboles en 12 millones de acres de la selva amazónica. La sequía y la escasez de agua son los problemas que más afectarán a los países del Tercer Mundo en la primera década del siglo XXI. De igual forma, una quinta parte de la humanidad no podrá obtener agua potable. (p.19)

Este panorama, determinante de todas las posibilidades futuras de vida, que evidentemente refleja el caos a que ha conducido el modelo político económico que ordena al mundo actual, este estado de cosas, es el que convoca la mirada del paradigma socio crítico, dado que su esencia le permite auscultar las razones sustanciales que han desencadenado una crisis que inevitablemente envuelve a lo social y con ello los componentes ético, político y valorativo. La implicación de estas dimensiones, no solo deberían generar reacciones de indignación y duros cuestionamientos a los parámetros morales que guían a nuestra sociedad, sino que además podrían motivar búsquedas de propuestas, sólidamente argumentadas De Souza, (2006). Esta postura teórica, necesariamente involucra el escrutinio a la razón instrumental subyacente a esta realidad y que ha dado lugar a estos grandes malestares, donde no solo sobresale la forma equívoca en la que se ha pretendido conducir y uniformar a las sociedades al interior de ciertos parámetros, sino que devela el conflicto que enfrenta la razón sustantiva dentro de ese razonar humano que se ha 
visto absorbido por los encantos superficiales de tales cánones, cánones que se han anclado en la conciencia colectiva gracias a los discursos hegemónicos que anulan alternativas emergentes que quizá gozan de mayor coherencia.

En estas circunstancias, es Habermas, como otro digno integrante de la teoría crítica, quien tiende un puente articulador entre las orillas de las dos mencionadas razones y descubre entonces la racionalidad de la acción comunicativa que es construida por los individuos o grupos humanos en el mundo cotidiano de la vida, a partir de aquellos intereses que emergen de las necesidades propias de la constitución humana y, que se han moldeado según las circunstancias socio históricas que atraviesan los conglomerados. Dentro de esta perspectiva, se diferencia la dimensión técnica, asociada al vínculo productivo y reproductivo que los sujetos establecen con la naturaleza, y la dimensión social, inmersa en la red de relaciones y normas que construye el hombre como sujeto cultural. Alvarado, \& García, (2008).

En esta atmósfera antagónica es que Habermas lanza su crítica al sistema técnico (instrumental) de la actual sociedad, que ha terminado devorando sin compasión al mundo de la vida, espacio éste en el que fluyen ideales como el de la igualdad, la libertad, la paz, la democracia, entre otros, que van concatenados con el equilibrio medioambiental. La oposición que la sociedad contemporánea enfrenta por la preeminencia de la cuestión instru- mental frente a los nobles ideales de la naturaleza humana, es el ángulo que lleva a Habermas a entender que aun dentro de lo diverso, de las contradicciones o rivalidades presentes en el mundo de la vida, es posible construir consensos promotores de emancipación y ello puede darse gracias a elementos simbólicos que comparten las sociedades, cuya naturaleza democrática puede llevar al entendimiento y la cooperación entre los individuos y los colectivos.

El interés emancipatorio que es la puerta de entrada a las posibilidades de liberación, no sólo se esfuerza por comprender el mundo, sino que también se esmera en transformarlo a partir de la generación de condiciones de autonomía racional y libertad que faciliten a los sujetos aportar a la consolidación de esos cambios. Desde esta perspectiva Habermas enriquece los postulados de la teoría crítica en la medida que impulsa el sentido autorreflexivo que sumerge al sujeto en el examen de las causas de su enajenación. De aquella meditación, muy posiblemente vienen en su auxilio las herramientas conducentes a erradicar las situaciones que lo alienan en los planos material y espiritual.

Este discurso, llevado a la problemática que atraviesan las comunidades por el cada vez más escaso recurso hídrico, es pertinente para comprender y reorientar las mentalidades y acciones del hombre frente a las dificultades que implican carecer del líquido vital, toda vez que desde este foco se pueden analizar las causas de la pro- 
blemática, elaborar diagnósticos y pronósticos, identificar las contradicciones de la racionalidad que motiva su mal uso, al tiempo que se formulan e implementan acciones que convocan a los actores sociales a desmoronar aquellos discursos y comportamientos causantes de la disminución hídrica.

Otro referente importante dentro de la teoría socio crítica y su vinculación a la problemática del agua es Foucault, a quien se trae a colación, porque la importancia estratégica de este preciado líquido, la ha llevado a estar en medio de un poder totalizante, de un autoritarismo económico que, aunque ha tenido manifiestos de resistencia, hasta ahora un grueso de los sectores sociales solo ha llegado hasta ahí, hasta las expresiones de inconformidad frente a un modelo hegemónico que en planteamientos De Sousa. (2006), remitiéndose a Foucault enuncia, “... en este $<<$ régimen de la verdad $>>$ no existe ningún escape emancipatorio frente al mismo, ya que la resistencia misma se ha convertido en un poder disciplinario y, por lo tanto, en un modo de opresión aceptada, internalizada" (p.22). No obstante, Foucault no se quedó en el plano de analizar los sistemas de poder que atrapan, cercenan, reprimen o normalizan, -aunque entendió que el poder no es neutro ni inocuo porque, siempre lo persigue la constante tentación de servir a determinados intereses-, su labor filosófica se extendió, de acuerdo con De Sousa, (2006), a darle "...credibilidad a la tarea de buscar «regímenes de la verdad» alternativos, de identificar otras formas de conocimiento que han resultado marginadas, suprimidas $\mathrm{y}$ desacreditadas por la ciencia moderna”. (p.22).

Desde este ángulo, y en diálogo con la perspectiva habermasiana, es que este trabajo pretende socavar el poder de una acción comunicativa que desde la racionalidad técnica ha promovido la insensata destrucción de los recursos naturales. Los intereses que guían la estructura de los conocimientos sociales están sujetos a los cánones de una cultura materialista viciada por la producción, distribución y acumulación de bienes, que no respeta los límites, que expone a la extinción a numerosas especies vegetales y animales, que evade y/o manosea la multiculturalidad, que fomenta e incrementa la miseria humana, que contamina y extingue las fuentes de agua y que no se detiene aunque se prevé la aproximación de un gran colapso como consecuencia del sobre abuso a que ha expuesto al medio ambiente en general.

De este mismo diálogo participa Edgar Morín (1999), con su teoría del pensamiento complejo, desde cuyo ángulo, aporta elementos ideológicos interesantes a la comprensión del tema que se viene abordando, en el sentido que la complejidad, tiene por imperioso lo dialógico, la comprensión, la democracia, como recursos que facilitan la aproximación "al sentido de las palabras del otro, de sus ideas, de su visión de mundo" (p. 52). Este enfoque amerita el desarrollo de unas habilidades y procesos de pensamiento abiertos, que conlleven, como plan- 
tea el referido autor "a respetar en el otro, a la vez, tanto la diferencia como la identidad consigo mismo" (p. 59) y esta exigencia, es apenas un mínimo necesario para iniciar la intervención de una temática que genera contradicciones alrededor del repertorio de discursos que están a favor de adelantar acciones en torno al cuidado y preservación de agua y aquellas posiciones para las que es indiferente la problemática, porque se han creído la falacia acerca de la abundancia generalizada del líquido en Colombia.

Bajo este estudio, subyace de manera discreta la mirada profunda del paradigma de la complejidad, cuyo abordaje no lineal de la realidad, parafraseando a Beberousse, (2008), obliga a ver los fenómenos como parte de un todo estructurado, donde los elementos se van a ver condicionados por las dinámicas que ocurren al interior y al exterior del sistema. Estas nociones aplicadas al tema en cuestión, contribuyen a analizarlo desde sus vínculos con la historia y la cultura, sus interconexiones con la economía, la política y educación, sus relaciones con lo subjetivo y lo intersubjetivo, con las esferas pública y privada, con la razón instrumental y la emancipatoria, es decir, el asunto del agua vinculado a una compleja red de relaciones que justifican la forma como se usa este recurso en cada sociedad.

En este sentido, Beberousse, (2008), interpretando a Morín, plantea: "La complejidad nació de la interacción de las partes que la componen, es decir, la complejidad se manifestó en el sistema mismo" (p. 99), por ello, cuando se consideran referentes asociados al agua, deben percibirse articulados a los diferentes componentes del sistema social, solo así pueden entenderse en su completa dimensión; de lo contrario seguiremos acostumbrados a satisfacer nuestras inquietudes con nociones superficiales, con discursos volátiles que carecen de asideros, pero que han generado estructuras mentales ordenadoras de modelos explicativos, que de manera elemental nos aproximan a realidades simplificadas $\mathrm{y}$ fragmentadas y, en efecto, desconectadas de la complejidad que les otorga sentido, fundamento y consistencia.

Así, las visiones de Sousa, de Habermas, de Foucault y de Morín, que beben de las fuentes de la teoría crítica, aportan elementos que desarrollan puntos de encuentro dentro de los tratados que consideran el recurso hídrico como un tema que involucra las desacertadas promesas de desarrollo del sistema moderno, la racionalidad de la acción comunicativa, los regímenes de la verdad inevitablemente asociados a la política y al poder y la evidente complejidad de una cuestión que involucra diversidad de actores y factores entre los que la educación desempeña un papel definitivo.

En este orden de ideas, abordar la educación como un elemento determinante de cualquier proceso formativo, necesariamente conduce a conectarla con el término pedagogía, ciencia que observada desde la tradición crítica permite comprender, según los referentes de Dilthey estudiados por Vás- 
quez, (2012) "porqué la educación no es propiamente un quehacer técnico, ni tampoco un dejar crecer sino un introducir al educando en el mundo de sentido, de valores, del deber ser" (p. 10). La educación entonces, al ubicarse en el campo del deber ser, desde el enfoque kantiano, es decir, en la esfera de la cultura, en el terreno del espíritu, acude a las prácticas de una razón superlativa para diferenciarse del mundo natural. Así, desde el enfoque de Dilthey, interpretado por Vásquez, (2012), la pedagogía es un constructo filosófico que le ha dado cuerpo teórico a la educación y su extensión práctica se dirige a "la bildung, la configuración del hombre, el autodesarrollo del espíritu, con exclusión de las exigencias y finalidades dadas por la naturaleza del hombre" (p.10) La bildung de Dilthey, se asocia a la educación como un producto de la cultura, cuya estructura se ha ido configurando a partir de un trascendente proceso histórico cultural, que influye y se arraiga en épocas y sociedades o que simplemente, por su grado de significancia, alcanza impactos importantes en su entorno de emergencia.

Esta acotación resulta coherente con los fines que en teoría persigue cualquier sistema educativo que se haya consolidado al abrigo del ideal ilustrado, más aún cuando éste, como parte de las ciencias sociales o humanas, según los lineamientos curriculares del Ministerio de Educación de Colombia, se erigió en parte fundamental de un proceso cultural notablemente comprometido con la expansión del proyecto moderno. Con relación a lo dicho, Pérez (1997, p. 48), agrega “... la escuela es una conquista social de la era moderna y tanto en su estructura como en su funcionamiento se encuentra adaptada a las exigencias sociales, políticas y económicas de aquella época”. Desafortunadamente, cuando el modelo educativo prusiano se pone al servicio de la revolución industrial para entrenar individuos que le sirvan al sistema productivo, el ideal Kantiano de una educación asociada a la emancipación ${ }^{1}$, pierde su rumbo y se convierte en un espacio de alienación, por cuanto su tarea se ha limitado a reproducir pautas de socialización enmarcadas en los cánones culturales del contexto, a entrenar talento humano para orden empresarial y a enmendar, en alguna medida, las consecuencias del desequilibrio socio económico.

En este contexto, los fundamentos de la educación, como la operatividad de la misma escuela, aparecen absorbidas por las exigencias mercantiles del capitalismo y segregadas de las cuestiones importantes de la sociedad. Ella (la educación), que tiene el deber superlativo de subordinar a su especialidad el conjunto de saberes afines a su quehacer, para congregarlos en una unidad, hoy, la episteme de la educación y su sustrato filosófico parecen experimentar una galopante exclusión de los grandes debates y de

1 Vázquez (2012, p. 11), comenta que en una entrevista de 1969 Theodore Adorno (1998) refiere este concepto a un trabajo de Kant en el que éste define la emancipación como la salida de la minoría de edad y pone como su condición la ilustración 
las políticas públicas. La explicación a ese fenómeno puede devenir de la reflexión planteada por Areyuna, \& Zúñiga, (2009), cuando aseguran que:

La desarticulación de lo público y de los proyectos nacionales desubicaron a la escuela, la dejaron sin lugar propio en el entramado social, esto sin olvidar que fueron los Estados nacionales los que la catapultaron a la cima de su apogeo, y los Estados actuales, atomizados y retirados de este campo de fuerza, son los que la dejan a la orilla del camino. (p.17)

Ese abandono a priori de la importancia de la escuela por parte del Estado, el derrumbe de la hegemonía que esta institución ejercía como transmisora del saber, la falta de empoderamiento y cimentación de su piso filosófico y como diría Narodowski (1999) citado por Areyuna y Zúñiga (2009), la declinación de la fuerza del discurso pedagógico moderno, conforman las grandes causas que han promovido la crisis de la escuela.

De otra parte, si se observa la educación desde la vertiente humanista, el hombre goza de facultades para reorientar actos y costumbres que puedan degradar su naturaleza y en consecuencia afectar el desarrollo de su plenitud. En este ambiente, la educación desempeña un papel trascendental, puesto que, al actuar sobre la naturaleza integral del sujeto, o bien sostiene la tradición, si se desarrolla una educación conservadora, o por el contrario, promueve la transformación de individuos y colectividades, si lo que se quiere es promover la apertura, el cambio, la liberación humana de la "conciencia oprimida", que en la perspectiva de Freire (1970), solo se logra superando el miedo a la libertad.

Ahora, si la educación, según la visión Freiriana, ha sido un instrumento de marginación y exclusión en manos de los centros de poder, una educación con rostro humano, debe atinar a formar una conciencia crítica en el individuo respecto a su ser, o sea, a sus potencialidades y limitaciones y, en lo concerniente a sus condiciones materiales, es decir a su cultura y a sus posibilidades socioeconómicas. De este modo, la educación se convierte en una aliada del desarrollo, del bienestar, o si se quiere, de la generación de una mejor calidad de vida. Sin embargo, el término desarrollo no puede tomarse a la ligera, cuando se opta por una educación emancipadora, pues no siempre el progreso se promueve como un bien que se reparte equilibradamente para todos.

Pero, como la educación requiere viabilizarse a través de normas e instituciones que hacen posible su concretización en el seno de los grupos humanos, fue oportuno inventar la escuela que, en sus inicios, en la expresión de Bonder (2013), fue definida como una "una agencia socializadora en la que conviven en tensión, representaciones de género diversas; un ámbito de lucha, resistencia y creación de regímenes de verdad, de normativas, valores y prácticas que se consideran "legítimas", "normales" y "transgresoras" (p. 18). Es decir que la escuela, como el útero donde se engendra la formación de las sucesi- 
Suárez Cerón, E. (2021). Aportes de la Escuela Fenomenológica y la Teoría Crítica a la Comprensión de la Problemática Hídrica dentro del Escenario Escolar. Estudios Latinoamericanos, ISSN (Impr) 0123-0301- ISSN (en línea) 2665-329X, 48-49, 5-28.

vas generaciones, vive en permanente dialéctica; no obstante, de esas contradicciones poco se conoce, porque los mecanismos de control que operan en su interior, están hechos para reprimir a quienes renuncien a la uniformidad o, simplemente, porque los actores del proceso educativo incorporan, "naturalmente" la normalidad de esas pautas en el desarrollo cotidiano. En el amplio sentido del análisis Foucaultiano, el ámbito escolar se sostiene en torno a discursos selectivos coadyuvantes del orden social y la misma escuela se encarga de regular el acceso del sujeto a esas narrativas particulares, que se estandarizan para garantizar el control.

Las razones de Foucault son avaladas por la experiencia de las sociedades que han tenido que tolerar la imposición de modelos universales de pensamiento y comportamiento, portadores de discursos fabricados por élites, que con fines hegemónicos los han canalizado por la vía educativa. De este modo, los sistemas educativos apalancados en proclamas que se sostienen en los regímenes de verdad y poder, garantizan el mantenimiento de un estado de cosas que mantienen enajenado al individuo y a sociedades enteras.

El nuevo panorama socio cultural y político económico, gestado en el seno de la ética moderna, ha generado un ambiente caótico para el desempeño educativo, en el que Areyuna y Zúñiga (2009) perciben la escuela como una organización desorientada, devaluada y despojada de su papel fundamental como difusora de saberes. Hoy son los nuevos agentes tecnicistas quienes la atrincheran, para reemplazar su rol como entrenadores de la masa laboral.

Esta preocupante realidad enciende alertas debido a que la Escuela es el segundo espacio conductor de valores, comportamientos y saberes que señalan el rumbo de la humanidad. En consecuencia, si la Escuela como ejecutora de los procesos educativos es empujada a desentenderse de su papel como formadora integral, temas como la formación ciudadana y ambiental quedarían fuera de su órbita, por lo que es preciso analizar los modelos educativos estatales, para luego, desarrollar plataformas de aprendizaje que faciliten la promoción de la condición humana y con ella el fortalecimiento de lazos interculturales que motivan relaciones sociales más democráticas y por tanto más armónicas con esa otredad y el espacio físico que habita.

En este orden de ideas, es preciso retomar el primer indicador de los Derechos Básicos de Aprendizaje (DBA) programado por el Ministerio de Educación Colombiano (2017), para el grado noveno, que enuncia: "[El estudiante] Analiza la situación ambiental de los geosistemas más biodiversos de Colombia (selvas, páramos, arrecifes coralinos) y las problemáticas que enfrentan actualmente debido a la explotación a que han sido sometidos" (p. 42). En este sentido, es probable estipular que las relaciones que las comunidades plantean entre ellas y a su vez con los recursos naturales, determinan finalmente el manejo del recur- 
so hídrico. Así, la pluralidad de visiones que emergen en los pueblos sobre el entorno, están inexorablemente ligadas a la educación, a los planes de vida y a la percepción que tengan de su territorio como espacio constructor de identidad y de múltiples interacciones.

Dentro de esta realidad se tiene que Colombia, al ser una nación mestiza, multicultural y que aún goza de una gran riqueza natural, constituye también un sistema complejo que avanza hacia la interculturalidad y, en esta ruta es imprescindible el papel que desempeña la educación en la apuesta por la preservación ambiental y la cohesión social en medio de un escenario multicultural. El pregón de la declaración universal de los Derechos Humanos (ONU, 2017), que en sus dos primeros artículos reza: "todos los seres humanos nacen libres e iguales en dignidad y derechos y, dotados como están de razón y conciencia, deben comportarse fraternalmente los unos con los otros... sin distinción alguna de raza, color, sexo, idioma, religión, opinión política o de cualquier otra condición” (p.1), alberga el compromiso de vivir en fraternidad como humanos que tienen corresponsabilidad con el espacio vital.

Dentro de esta concepción, a la educación le compete liderar estrategias que promuevan el desarrollo de ideales como la paz, la libertad, la justicia social, el diálogo entre culturas, el cuidado y protección de recursos naturales como el agua y, para ello, el problematizar en el aula, la crisis por la que pasan dichos ideales, permite empoderar de razones y acciones a los educandos, a fin de que desarrollen responsabilidad social, sentido de empatía e inconformismo con hechos que representen el deterioro de la condición humana y que obviamente amenacen con la destrucción de esa casa común que es nuestro planeta. La misma Declaración, en el artículo 26 establece que:

"la educación tendrá por objeto el pleno desarrollo de la personalidad humana y el fortalecimiento del respeto a los derechos humanos y a las libertades fundamentales; favorecerá la comprensión, la tolerancia y la amistad entre todas las naciones y todos los grupos étnicos o religiosos y promoverá el desarrollo de las actividades de las Naciones Unidas para el mantenimiento de la paz". (ONU, 2017).

De manera que la educación apoyada en el currículo que también incluye los proyectos ambientales escolares (PRAE), tiene la gran responsabilidad de viabilizar la formación permanente de las personas y las sociedades en el marco de una serie de principios ambientales y valores humanos que coadyuven a construir armonía dentro de la pluralidad. En sintonía con esta apreciación, Rodolfo Stavenhagen (2016), miembro de la Comisión Internacional sobre la Educación para el siglo XXI, en otros términos, plantea que una educación con esencia multicultural debe tener la capacidad de responder a la constante actual, marcada por la interrelación global y nacional, pero sin descuidar las realidades locales que están impregnadas 
de culturas particulares. Este reconocimiento del mundo culturalmente diverso, es el punto de partida para fomentar la tolerancia y la aceptación de las diferencias, comenzando por el entorno próximo, con inmediata proyección a culturas distantes en tiempo y espacio.

Infortunadamente estas apreciaciones, solo pasan a ser unas indicaciones para el deber ser de una educación multicultural integral, pues según un estudio de la Comisión Económica para América Latina - CEPAL (2014), la transgresión a las pautas económicas tradicionales y su evolución a una producción extractivista en los territorios de las comunidades ancestrales, ha desembocado en tragedias ecosistémicas y en la reorganización espacial de las poblaciones y sus actividades como causas que hoy redundan en el despojo de sus derechos, la violación a sus cosmovisiones y la destrucción de su tejido social, panorama que en variados contextos, ha terminado mayormente degradado, por efecto del racismo, la marginalización socio económica y las tragedias ambientales que castigan con más énfasis a los sectores sociales más deprimidos.

La referencia a estas realidades se complejiza si se le añade fenómenos como el cambio climático, el crecimiento poblacional y la depredación descontrolada de los recursos naturales, como hechos que se perciben cada vez con mayor intensidad. Las fuertes temporadas de sequía en algunos lugares y las lluvias excesivas que producen inundaciones en otros, po- nen en riesgo la existencia de la vida en el planeta y si bien son temas que alimentan la inspiración de periodistas, que día tras día llenan centenares de columnas con las aflicciones que enfrenta la humanidad, como consecuencia de la afrenta que el hombre le ha declarado a la naturaleza, los tecnócratas que formalizan los proyectos escolares, insinúan la importancia de estos temas, pero descuidan las estrategias de participación de la comunidad como generadora de la problemática, como receptora de las consecuencias y como promotora de soluciones.

En este sentido, puede tomarse como ejemplo el tema del agua, que en Colombia suele aparecer como un asunto que reviste importancia cuando asoman temporadas en las que amenaza la presencia de fenómenos climáticos como el niño o la niña. Los noticieros del país también acostumbran a difundir las alertas de la ONU sobre la problemática del agua en el mundo; sin embargo, la realidad muestra que la cuestión del recurso hídrico sigue siendo un problema que no se ha considerado con la suficiente seriedad y compromiso, tanto por los administradores de este bien o sea el Estado, como por los usuarios del mismo, es decir la comunidad.

Estos desequilibrios cada vez más frecuentes necesitan de políticas gubernamentales comprometidas que contrarresten la crisis ambiental que se vive. Como se percibe a simple vista, en épocas pasadas se disponía de agua en abundancia para el trabajo agrícola, el consumo humano, para 
los animales y para las diversas actividades que realiza el hombre. En la actualidad, la disminución del agua en la naturaleza, genera dilemas que desestabilizan no solo al sistema político - económico sino a las comunidades y a cada individuo como tal. Lamentablemente, si en el reparto de estas consecuencias, los afectados son negritudes, indígenas, campesinos o comunidades marginadas, el tema no pasa más que ser noticia de un día.

La descripción de este crudo panorama bloquea en gran medida la concreción del ideal educativo multicultural, expresado por Rodolfo Stavenhagen, en la medida que las autoridades gubernamentales dan prioridad a las exigencias de un modelo hegemónico completamente desinteresado en los estragos que dejan sus mecanismos de producción, distribución y consumo. De ahí que entre en abierto choque, la visión de desarrollo que tienen los pueblos autóctonos forjados en sus planes de vida, que se canalizan hacia el "buen vivir" y aquella perspectiva acumulativa, materialista y arrogante de la corriente capitalista, que solo se orienta a incrementar la riqueza y el poder de un reducido sector de la sociedad, mientras deja por doquier estelas de pobreza, violencia, desigualdad, opresión, injusticias, contaminación, miseria, caos ambiental y destrucción de la vida en general.

Por ello es que es importante problematizar estas realidades desde el Aprendizaje Basado en Problemas (ABP) y promover cambios en la comunidad educativa a partir de la arti- culación de las prácticas pedagógicas creativas y de las competencias ciudadanas al Proyecto Ambiental Escolar (PRAE), aunque de antemano se sabe que el poder del modelo económico representado en las transnacionales y su peso en el sistema de gobierno, sigue aferrado a un neocolonialismo que a todas luces no le interesan los aportes de la multiculturalidad y, por ende, la inversión en educación para adecuar sus estructuras al desarrollo de los valores, tradiciones, cosmovisiones y en fin, lo que sería la promoción de la interculturalidad, no es parte de las prioridades del orden político económico vigente en nuestro Estado.

En este sentido y al aproximarnos al tema del PRAE, hay que precisar que el grueso de la educación ambiental en Colombia gira en torno a este proyecto. En la mayoría de instituciones educativas estos proyectos pedagógicos que motivan el estudio y el discernimiento de los problemas y el patrimonio ambiental del contexto y la región, quedan a cargo del área de ciencias naturales; en muchos casos, sin la transversalidad necesaria, sin adecuarse a la localidad, sin involucrar a la comunidad educativa y como si fuera poco, se suelen quedar solo en la etapa de planeación o en la mera tarea de transmisión de datos y conceptos sobre el medio ambiente o en la conmemoración de fechas insignes sobre el mismo tema. Este tipo de prácticas que ha ido haciendo carrera en el país, ha determinado el bajo porcentaje de PRAEs que han llegado a consolidarse como experiencias significativas. 
En términos de lo que se ha venido trabajando en este documento y como el foco es el recurso hídrico, que conforma uno de los ejes del PRAE, la educación ambiental necesita extrapolarse al contexto, mediante una mecánica que aperture espacios de participación que den opción a implementar soluciones afines con las dinámicas naturales y socioculturales locales. Este enganche es necesario para forjar diálogos comunes entre estudiantes y comunidad, si se pretende empezar a crear conciencia colectiva. En este sentido, es válido rescatar el aporte de Bonilla, (2015), que asume "La educación [como] un proceso permanente y altamente complejo que se da en todos los escenarios en que existe interacción social y a lo largo de la vida..."(p. 41). Esta forma de ver la educación, necesariamente desemboca en percibir lo ambiental y educativo como un sistema en constante interacción, en permanente feedback. Desde este ángulo es que llega a comprenderse que la problemática ambiental es la manifestación de la crisis civilizatoria, expresada en unas pautas culturales de origen occidental, que han justificado el dominio insustentable de la naturaleza.

Bajo esta mirada y percibiendo lo cultural como un asunto complejo y sistémico, la Educación Ambiental llega a comprenderse, según Paz, Avendaño, y Parada, (2014), como “... un proceso de reproducción y transformación cultural, ... en torno al cuidado y uso racional de cada uno de esos elementos que integran el medio ambiente, para no arriesgar las condiciones requeridas para una vida digna” (p.6). Este referente, de algún modo es compatible con el décimo fin de la ley 115 de 1994 (Ley General de Educación de Colombia), referido a la sustancia de lo que luego orientara el sentido de los PRAE, esto es "la adquisición de una conciencia para la conservación, protección y mejoramiento del medio ambiente, de la calidad de vida, del uso racional de los recursos naturales [...]". Es evidente que el espíritu de este propósito comulga con preocupaciones del desarrollo sostenible, que busca fomentar el uso sensato de los recursos en el presente, para hacer sostenible su gestión y heredad a las generaciones futuras. Pero esta acción, aparentemente elemental, convoca la puesta en marcha de todo un sistema de valores sociales que reorienten la visión de desarrollo y de proyección futura de las comunidades; emprendimiento éste que amerita la articulación de la educación a la esfera ambiental, en función de formar ciudadanos capaces de manejar relaciones armónicas con espacio natural y de tomar decisiones solidarias con el bienestar colectivo. La tarea es de tanta envergadura, que el Ministerio de Educación Nacional de Colombia MEN considera que “... lo ambiental y la educación ambiental se relacionan directamente con la construcción de un proyecto de sociedad". (Ministerio de Educación Nacional de Colombia MEN 2004, p. 1)

Sin embargo, Leff (2017) aclara que “... la educación ambiental no ha conseguido transformar los regímenes educativos institucionales en América 
Latina y sigue siendo marginal dentro de las prioridades de la comunidad educativa" (p. 158). Si se relaciona el aporte de Leff con las anotaciones anteriores, su juicio es pertinente, por cuanto los PRAE no se están aprovechando en las aulas como estrategias pedagógico - didácticas interdisciplinarias, que permitan indagar y reflexionar críticamente sobre la realidad ambiental, ni como mecanismos metodológicos que ayuden a canalizar acciones de intervención que comprometan la participación de los distintos actores que conforman la comunidad educativa, con la consecuente proyección comunitaria.

Ahora, respecto al recurso hídrico, que constituye un eje fundamental del PRAE, es común escuchar que es un elemento indispensable para la vida, que sin el todo ser vivo estaría condenado a desaparecer, que el agua es fuente de todo tipo de vida, que nuestro cuerpo y el planeta, alrededor del $70 \%$ son agua. Es decir, el agua tiene tanta importancia para las funciones básicas de la vida que no solo debe tratarse como un patrimonio de la humanidad, sino que su uso debería ser mucho más democrático. Empero, las determinaciones del actual modelo de mercado, han llevado a cosificarla y a trasladarla al lenguaje comercial, como una mercancía cuyo valor está determinado por la racionalidad económica capitalista, que no se molesta en cuestionar las acciones patrocinadas desde su proyecto y que están socavando las bases de sustentabilidad de la vida.
Dentro de estos rasgos característicos de las sociedades modernas campea la ignorancia sistemática sobre los impactos que causa la economía industrial en el escenario ambiental. Dicho modo de producción se ha declarado enemigo del cuidado y conservación de los recursos naturales, de ahí que Márquez (2003), lo califique como "ecológicamente depredador, socialmente injusto y económicamente inviable, es decir insostenible" (p.13).

La dura realidad antes comentada se resume en una estructura socio cultural y político económica contemporánea, signadas por el despojo del agua a sectores pauperizados de la humanidad. Ese secuestro del recurso hídrico viene de la mano de factores como la contaminación, el desvío de cauces, la construcción de mega obras como túneles y presas hidroeléctricas, la actividad minera a gran escala, la deforestación, abiertamente patrocinados por multinacionales aliadas de los gobiernos, cuyas acciones premeditadas van dejando estelas de miseria y orfandad en las regiones y áreas que ambientalmente van sufriendo una degradación y desertización que no tienen dolientes y, que a la postre generan desplazamientos, enfermedades y hambrunas que complejizan el deterioro ambiental.

Esta cuestión tiene claras raíces en la modificación de los patrones que rigen en las actuales actividades productivas, entre los cuales se incentivan conductas desperdiciadoras y abusivas del gasto hídrico, así como hábitos contaminantes y destructores 
de los ecosistemas, en donde los humedales son los que han sufrido los peores castigos de la acción humana. El detrimento de estos hábitats constituye una pérdida riesgosa del patrimonio natural que va ligado a pérdidas culturales que se evidencian en el creciente deterioro de los saberes populares, especialmente aquellos que se anclan en las prácticas de la ruralidad, generalmente asociadas a las formas de aprovechamiento del suelo. (Márquez, 2003)

Con razón Koichiro Matsuura, director de la UNESCO para el año 2003, con motivo de la publicación del Informe sobre el Desarrollo de los Recursos Hídricos en el Mundo, expresó que "De todas las crisis sociales y naturales que debemos afrontar los seres humanos, la de los recursos hídricos es la que más afecta a nuestra supervivencia”. Tales términos recogen las conclusiones de estudios estadísticos con pronósticos sombríos para una humanidad cuya existencia y prolongación están determinadas por este líquido vital.

Por ello es que se hace urgente adelantar procesos pedagógicos que aterricen al aula las directrices que han emitido organismos internacionales para atender la crisis del agua y que algunos Estados como el colombiano, han traducido en políticas y programas que pretenden aliviar o contener los efectos del manejo irracional de los recursos hídricos. Uno de esos lineamientos es la Política Nacional de Gestión Integral del Recurso Hídrico (PNGIRH). El sexto objetivo de esta política está consagrado a la gobernabilidad dentro de la cual se hace énfasis en la participación y la cultura del agua, que pretenden impulsarse desde líneas estratégicas asociadas al desarrollo de campañas educativas que sensibilicen sobre las acciones integrales de manejo del agua, así como la articulación de contenidos curriculares contextualizados, que vinculen los propósitos de la política en mención.

De esta forma, esa necesidad que percibe Leff, respecto a que la educación ambiental transforme los sistemas educativos institucionales, puede viabilizare a partir de una mirada fenomenológica del asunto, por cuanto la referida escuela como partera de la comprensión de las experiencias de los sujetos asociadas a un fenómeno, genera estrategias didácticas que inducen la capacidad de asombro como antesala de aquellas preguntas que inquietan la conciencia y que perturban la percepción del "orden natural" al que los sujetos se acostumbran. Dicha comprensión, que acontece como derivado de procesos metacognitivos, disciernidos en la conciencia, que decanta sus aprendizajes en el fragor de las experiencias colectivas, es factible gestionarse a partir de herramientas metodológicas como el Aprendizaje Basado en Problemas - ABP, cuya lógica deductiva, nacida de la mayéutica, conduce a desentrañar las causas de las razones, a inquirir el origen de esas causas, a analizar los efectos que producen, hasta lanzar al sujeto a proponer alternativas que se han gestado en su aprendizaje autónomo y que luego se traducirán en habilidad discursiva, 
en acción comunicativa emancipadora de la conciencia. De esta manera, la fenomenología, como escuela que surgió de la preocupación por explicar la naturaleza de los hechos, genera recursos metodológicos para aprehender la realidad desde el aula como escenario que da lugar a reconstruir, retroalimentar, descartar o validar el conocimiento.

De esta manera, el Aprendizaje Basado en Problemas - ABP, se presenta como una de las tantas estrategias pedagógicas utilizadas para dinamizar el trabajo en el aula y del cual, la Universidad Politécnica de Madrid (2011), conceptualiza: "Es una metodología centrada en el aprendizaje, en la investigación y reflexión que siguen los alumnos para llegar a una solución ante un problema planteado por el profesor" (p. 4). Los términos investigación y reflexión abocan al estudiante a enfrentar la incertidumbre como estrategia develadora de las cegueras del conocimiento. De acuerdo con Morín, E. (1999) esas obcecaciones del saber nos han creado una seguridad garantizada, que ha forjado estructuras mentales sin capacidad para incorporar los conocimientos recientes que aparecen con frecuencia y de manera espontánea. Esa nueva información requiere un formato cognitivo de enganche, que facilite su asimilación a las estructuras previas, condición ésta que conduce a generar los aprendizajes significativos.

De este modo, la investigación y la reflexión se convierten en herramientas valiosas que conducen a revalorar los saberes aprehendidos y despliegan la posibilidad de crear soportes conceptuales que facilitan el anclaje de nuevos conocimientos sobre la base de una estructura cognitiva renovada. Esta es la gran tarea del ABP, de lo contrario, los nuevos saberes y experiencias podrían malograrse al intentar asociarse a los viejos paradigmas. De ahí que las dos habilidades mencionadas, sean determinantes a la hora de asumir e internalizar los cambios que llegan, pues sin este ejercicio, pretender ingresar con éxito a aquellos saberes fortuitos en estructuras mentales viciadas por la ceguera, constituirá un desgaste que no impactará en las trasformaciones que se quiere lograr.

A estas disertaciones en torno al ABP, se agregan el desarrollo de la capacidad autónoma que alcanza el alumno implicado en la resolución de preguntas, problemas y/o procesos investigativos y el avance en el trabajo cooperativo entre docentes y estudiantes que busca atender a situaciones problematizadoras de la vida cotidiana, en donde se conjugan la adquisición de conocimientos, la formación en actitudes, valores y se hacen aportes que contribuyen a mejorar el bienestar del grupo, del colectivo, de la institución o de la misma comunidad.

Desde este escenario, al ABP le interesa tratar las realidades que tocan las fibras humanas, las percepciones pertinentes al mundo de la vida, lo cotidiano, lo que envuelve o afecta las acciones de individuos y colectivos. De esta manera, según Morín, E. (1999), "El conocimiento de los problemas claves del mundo, ... debe ser tratado so pena de imperfección cognitiva, 
más aún cuando el contexto actual de cualquier conocimiento político, económico, antropológico, ecológico... es el mundo mismo" (p.14). Así, dejar conducir la labor pedagógica por las bondades del ABP, promueve la educación en contexto y el desarrollo de una inteligencia integral, en la medida que para abordar los problemas necesita integrar los saberes, lo multicultural, las normas, los valores, los miedos, las esperanzas, es decir, tener en cuenta la condición humana en sus encuentros y desencuentros, en sus conflictos y reconciliaciones, pero también en la riqueza de su diversidad.

Así, al interior de toda esta riqueza pedagógica que despliega el ABP, es pertinente reconocer la importancia de los conocimientos previos, pues son como las nociones rudimentarias que guían el proceder de los estudiantes, que son susceptibles de sufrir reacomodaciones a través del anclaje de ideas y conceptos que tienen significancia para el individuo; luego, la información que es mejor asimilada, es usada para resolver las controversias que ocurren en la rutina de la vida. Así, Llorens, refiriéndose a este planteamiento considera que "los conocimientos previos... determinan el volumen y la naturaleza de lo que el alumno va integrando posteriormente en su proceso de aprendizaje, siendo continuamente activados y reestructurados a medida que se avanza en el análisis y resolución del problema". (Llorens, 2010, p. 2).

Obviamente, las reacomodaciones del conocimiento que ocurran en lo sucesivo, dependen de la manera de enfrentar las incertidumbres, para lo cual según Morín (1999), es preciso “... enseñar principios de estrategia que permitan afrontar los riesgos, lo inesperado, lo incierto, y modificar su desarrollo en virtud de las informaciones adquiridas en el camino" (p.3). Mas adelante para complementar su propuesta agrega: "Es necesario aprender a navegar en un océano de incertidumbres a través de archipiélagos de certeza”. Tales archipiélagos estarán formados por los conocimientos previos y los océanos serán los mares de posibilidades que se abren frente a la solución de un problema.

En este orden de ideas, el ABP por la flexibilidad que representa para abordar en el aula los dilemas que afectan la vida del estudiante, es ideal para introducir los conversatorios, el debate, la reflexión y en general, la deconstrucción y construcción de perspectivas colectivas sobre el manejo del recurso hídrico y las maneras de promover el uso adecuado del mismo. Necesariamente, esta iniciativa debe estar inserta en el Proyecto Ambiental Escolar (PRAE) a fin de institucionalizar procesos que logren socavar los fundamentos de costumbres insanas, cuyos efectos empiezan a sentirse con rigor gracias a los largos decenios de vulneración a los recursos naturales.

Finalmente hay que agregar que el ABP constituye una posibilidad significativa en la construcción y adquisición de conocimiento en los espacios escolares; así mismo, este tipo de metodología ofrece la oportunidad de generar un aprendizaje activo, donde el estudiante aprende haciendo y los profesores, desde la didáctica de la 
mayéutica, estimulan en el alumnado la generación de nuevas opciones para atender las problemáticas que sean de la preocupación de la clase, pues si se tiene en cuenta este factor, se facilita la integración de los nuevos conocimientos a los saberes previos y desde ahí se posibilita una mayor retención al canalizar la transferencia del mismo a otros contextos. Además, la adquisición de habilidades cognitivas, principalmente relacionadas con el razonamiento, la reflexión y el desarrollo de capacidades propositivas para la solución de problemas da lugar al aprendizaje autónomo; adicionalmente también ayuda al desarrollo de competencias y habilidades sociales como el trabajo en equipo, la comunicación efectiva, el respeto hacia los demás y la responsabilidad ética con el entorno.

Para concluir, es importante señalar que los aportes de la escuela fenomenológica y de la teoría socio crítica residen en el fortalecimiento de la educación humanista, en cuya esencia el hombre goza de facultades para reorientar sus actos y costumbres e incluso impactar en las colectividades, mediante la apertura, el cambio, la liberación humana de la "conciencia oprimida", que en la perspectiva de (Freire, 1970), solo se logra superando el miedo a la libertad. Freire, que sería una de las figuras más representativas de las teorías críticas de la educación, percibe que el escenario escolar no es ajeno a las realidades comunitarias en las que inserto, pues las convulsiones sociales, el deterioro ambiental, la contaminación hídrica, el cambio climático, las relaciones de poder, la miseria, las diferencias ideológicas, las aspiraciones, las esperanzas, en fin, cada universo particular, que ingresa al aula, lleva consigo diversidad de problemáticas susceptibles de problematizarse con fines pedagógicos.

No obstante, hay que tener en cuenta que de la vertiente educativa y de la pedagógica emergen las prácticas de aula que visualizan la tensión entre las proyecciones del currículo y la cotidianidad del alumnado. Dicha resistencia emerge de la distancia que media los saberes curriculares y los métodos, desfase que fortalece las trincheras entre lo que se aspira y los distintos ritmos de actuación que asumen los agentes de la acción educadora. Herrera, (2013).

Esta apreciación que de entrada denota un conflicto entre la planeación escolar y el contexto socioeconómico de la población estudiantil y que además pone en evidencia el boquete entre los contenidos y los métodos y, entre las aspiraciones y la realidad, no es más que el reflejo de las tensiones entre los procesos mercantiles de la educación y las realidades cotidianas que sumen a las comunidades educativas, alienadas en un sistema político económico que ha conducido al relajo moral, al desconocimiento de la diversidad, a la deshumanización y al caos social y ambiental.

Frente a este escenario, la educación enriquecida con las contribuciones de la tradición crítica y como productora de formación integral, promueve acercamientos entre los diferentes actores del proceso y entre los saberes curriculares y la realidad y, se convierte en una aliada del desarro- 
llo, del bienestar, o si se quiere de la generación de una mejor calidad de vida, cuya riqueza supera los límites del individualismo, para ir en pos de la inclusión de los "otros", de la vida contenida en el medio ambiente, para en conjunto, forjar un tejido de corresponsabilidades, de armonía y equilibrio, que ayude a superar el dualismo humanidad - naturaleza.

\section{Referencias}

Alvarado, L., \& García, M. (2008). Características más relevantes del paradigma socio crítico: su aplicación en investigaciones de educación ambiental y de enseñanza de las ciencias realizadas en el Doctorado de Educación del Instituto Pedagógico de Caracas. Revista Universitaria de Investigación, 9(2), 187-202. Recuperado de https://www.redalyc.org/ pdf/410/41011837011.pdf.

Anzola, J. (2007). La fenomenología de la conciencia en E. Husserl. Universitas PhilosoPhica, 24 (48), 127 - 147, Bogotá, Colombia. Recuperado de https://dialnet.unirioja.es/servlet/articulo? codigo $=2873682$.

Areyuna, B., \& Zúñiga, J. (2009). Magíster en educación y cultura transformaciones contemporáneas y educación. Universidad ARCIS. Registro Propiedad Intelectual No 141.879. Libertad 53 / Santiago / chile

Bonilla, N. (2015). Aprender a vivir: una experiencia de educación ambiental en el Colegio Rural José Celestino Mutis. Universidad Nacional de Colombia.

Barberousse, P. (2008). Fundamentos del pensamiento complejo de Edgar Morín. En Revista Educare XII(2), 95-113, Universidad de Heredia Costa Rica.

Bonder, G. (2013). La equidad de género en las políticas educativas: Una mirada reflexiva sobre premisas, experiencias y metas. Revista Sinergias Cuadernos del Área Género, Sociedad y Políticas - FLACSO, Argentina. Recuperado de http://www.prigepp.org/media/documentos/ sinergias/Vol2_sinergias_educacion.pdf

Comisión Económica para América Latina (2014). Los pueblos indígenas en América Latina. Avances en el último decenio y restos pendientes para la garantía de sus derechos. Santiago: CEPAL. Recuperado de https://repositorio.cepal.org/bitstream/handle/11362/37222/ S1420521_es.pdf.

De Sousa, B. (2006). Conocer desde el Sur. Para una cultura política emancipatoria. Biblioteca nacional del Perú. Recuperado de https://estudogeral.sib.uc.pt/bitstream/10316/44158/1/Conocer\%20desde\%20el\%20Sur.pdf.

Freire, P. (1970). La Pedagogía del Oprimido. Siglo XXI editores. México, D.F. Recuperado de https://fhcv.files.wordpress.com/2014/01/freire-pedagogia-del-oprimido.pdf

Herrera, J. D. (2013). Pensar la educación, hacer investigación. Facultad de ciencias de la educación. Bogotá: Universidad de La Salle.

Lambert, C. (2006). Edmund Husserl: la idea de la fenomenología. Facultad Cs. Religiosas y Filosóficas Universidad Católica del Maule. Teología y Vida, 7(4), 517 - 529. Recuperado de https://scielo.conicyt.cl/scielo.php?script=sci_arttext\&pid=S0049-34492006000300008

Leff, E. (2017). Pensamiento ambiental latinoamericano: patrimonio de un saber para la sustentabilidad. En: El pensamiento ambiental del sur. Complejidad, recursos y ecología política latinoamericana. Compilador Walter Alberto Pengue. Ediciones UNGS, Universidad Nacional General Sarmiento. Buenos Aires Argentina 
Lozano, V. (2006). Husserl y la historia. Recuperado de ocplayer.es/40950872-Husserl-y-la-historia. html.

Llorens, J. (2010). El aprendizaje basado en problemas como estrategia para el cambio metodológico en los trabajos de laboratorio. En Quim. Nova, 33(4), 994-999. Valencia España. Recuperado de http://www.scielo.br/scielo.php?script=sci_arttext\&pid=S0100-40422010000400043.

Márquez, D. (2003). De la teoría a la práctica en los procesos de educación ambiental: el agua elemento dinámico. Congreso Agua y educación ambiental: nuevas propuestas para la acción. Alicante 26, 27, 28 Y 29 de noviembre de 2003. Caja de ahorros del Mediterráneo. Recuperado de http://www.ciceana.org.mx/web/recursos/tribunatura/pdf/Agua_y_educacion_ambiental.pdf

Ministerio de Educación Nacional de Colombia. (1994). Ley General de Educación. Recuperado de https://www.mineducacion.gov.co/1621/articles-85906_archivo_pdf.pdf.

Ministerio de Educación Nacional de Colombia. (2004). Educar para el desarrollo sostenible. Altablero, No. 36, agosto - septiembre 2005. Recuperado de https://www.mineducacion.gov. co/1621/article-90893.html.

Ministerio de Educación Nacional de Colombia. (2017). Derechos Básicos de Aprendizaje Ciencias Sociales. Recuperado de https://wccopre.s3.amazonaws.com/Derechos_Basicos_de_Aprendizaje_Sociales.pdf.

Morín, E. (1999). Los siete saberes necesarios para la educación del futuro. Organización de las Naciones Unidas para la Educación, la Ciencia y la Cultura. París.

Organización de Naciones Unidas. ONU (2017). Declaración universal de derechos humanos. Adoptada y proclamada por la Asamblea General en su resolución 217 A (III), de 10 de diciembre de 1948. Recuperado de https://www.un.org/es/sections/what-we-do/protect-human-rights/

Paz, L., Avendaño, W., \& Parada, A. (2014). Desarrollo conceptual de la educación ambiental en el contexto colombiano. Luna Azul ISSN 1909-2474. No. 39, julio - diciembre 2014. Recuperado de http://www.scielo.org.co/pdf/luaz/n39/n39a15.pdf.

Pérez, A. (1997). Socialización y educación en la época posmoderna. En Ensayos de pedagogía crítica. España editorial popular 1997 pp. 45 - 66.

Ropero, N. (2019). Teoría crítica y fenomenología: ¿una síntesis necesaria? Bases fenomenológicas para una revisión crítica del proyecto filosófico del primer Marcuse. En Enrahonar An International Journal of Theoretical and Practical Reason 62, 117-143. Recuperado de https:// dialnet.unirioja.es/servlet/articulo?codigo $=6849953$.

Stavenhagen, R. (2016). La educación para un mundo multicultural. Perseo Programa universitario de derechos humanos. Número 46 diciembre de 20216. Universidad Nacional Autónoma de México. Recuperado de: http://www.pudh.unam.mx/perseo/la-educacion-para-un-mundo-multicultural1/.

UNESCO (2003). Correo de la UNESCO. Informe sobre el Desarrollo de los Recursos Hídricos en el Mundo. Recuperado de https://es.unesco.org/courier/octubre-2003?language $=\mathrm{fr}$

Universidad Politécnica De Madrid. (2011). Aprendizaje Basado en Problemas, Guías Rápidas sobre Nuevas Metodologías. Recuperado de: http://innovacioneducativa.upm.es/guias/Aprendizajebasadoenproblemas.pdf.

Vásquez, S. (2012). La filosofía de la educación. Estado de la cuestión y líneas esenciales. CIAFIC ediciones. Recuperado de http://www.ciafic.edu.ar/documentos/FilosofiadelaEducacionVazquez_2da_Ed.pdf 